\title{
ANNXIETY DISORDERS
}

natuire DISEASE

REVIEWS PRIMERS

Anxiety disorders are a group of psychiatric conditions that affect one in four individuals. They are characterized by excessive fear, anxiety, the avoidance of perceived threats and, in some cases, panic attacks.

\section{EPIDEMIOLOGY}

General risk factors for anxiety disorders include female sex and a family history of anxiety or depression. Early life risk factors include a withdrawn or inhibited temperament, overinvolved or negative parental interactions, reduced peer relationships and physical or sexual abuse. In adults, several life stressors, such as family illness and relationship breakdowns, can contribute to the development of anxiety disorders.

\section{Ry MaMagaser}

Often, cognitive-behavioural therapy (CBT; a goal-orientated, skills-based therapy) is the first-line treatment for anxiety disorders. Other psychological therapies can include mindfulness and acceptance-based therapies and interpersonal therapy. Pharmacological treatment can be combined with psychological therapy, or can be used separately. Commonly used drugs include antidepressants, benzodiazepines, atypical antipsychotics and $\beta$-adrenergic blockers.

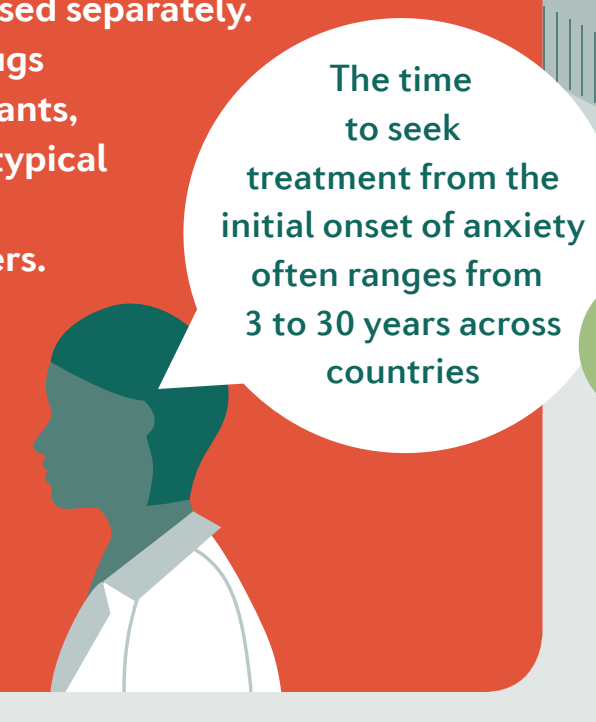

\section{The time} reatment from itial onset of anxiety

$\beta$ - often ranges from

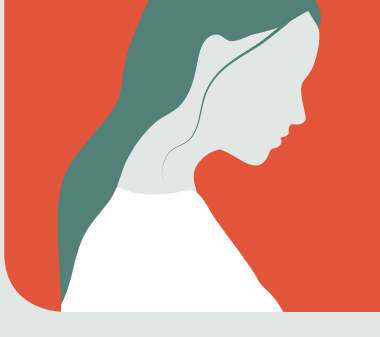

\section{Oi) OUTLOOK}

Further research to understand the precise risk factors, genetic factors and mechanisms of anxiety disorders is essential. This could also lead to the

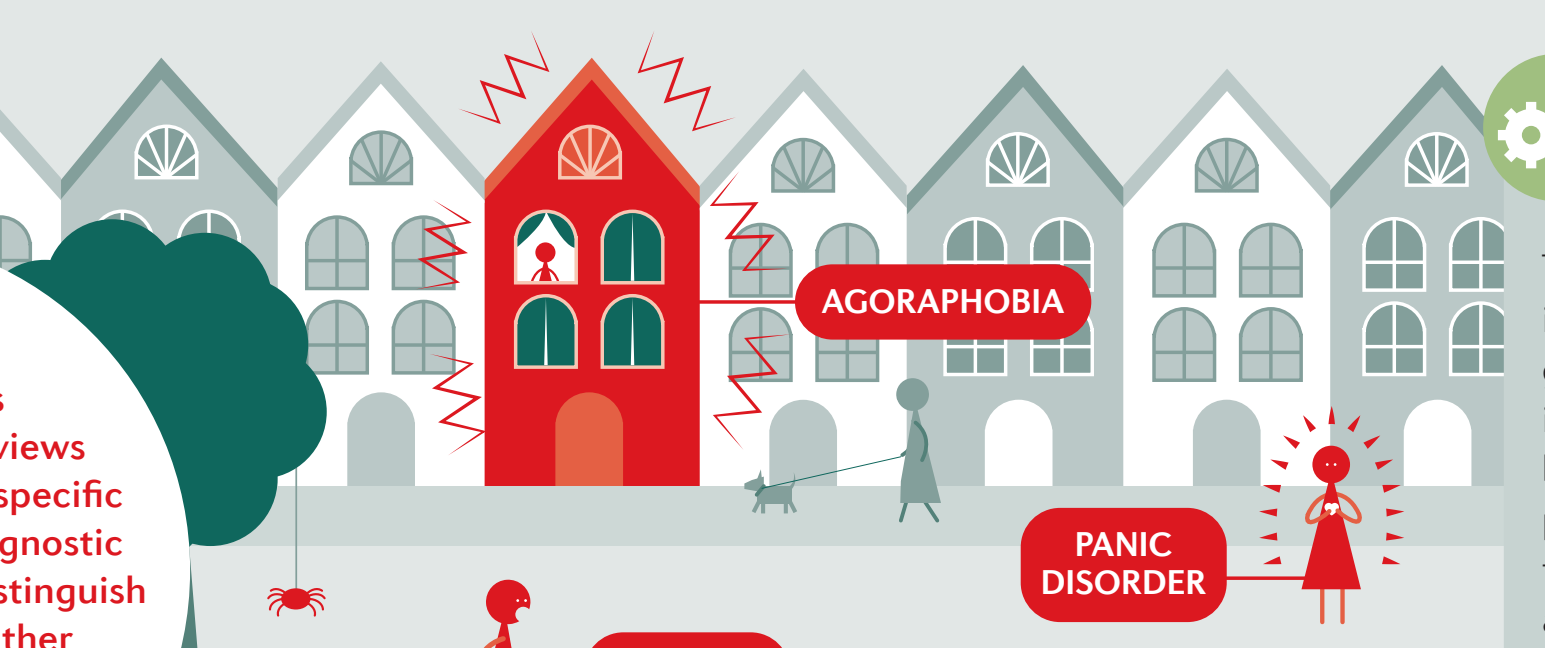

MECHANISMS

.

The neurobiology underlying the development of individual anxiety disorders is unknown, but some generalizations in the way anxiety is processed in the brain have been identified. Namely,

bidirectional connections between the amygdala, prefrontal cortex, anterior cingulate cortex and the hippocampus are thought to underlie fear and anxiety. Dysfunction of these areas, in addition to the hypothalamic-pituitary-adrenal axis, might contribute to the development of anxiety disorders. Twin studies have revealed moderate genetic and substantial environmental influences. Few specific genetic risk factors have been identified, but this is likely to increase in the future.
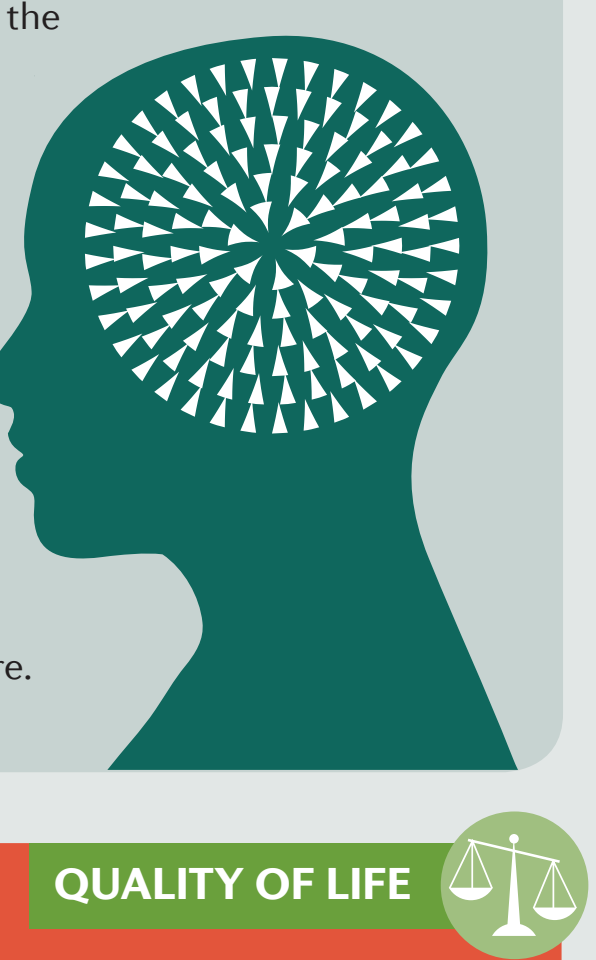

Anxiety disorders are one of the most persistent mental health disorders and, as such, can substantially reduce the quality of life of the patient. Regarding individual disorders, the disabilities and comorbid complications associated with panic disorder and generalized anxiety disorder are more severe than those caused by specific phobias.

development of biomarkers and new therapies that specifically target distinct alterations in patients with anxiety disorders.
$60-90 \%$ of patients with an anxiety - disorder have a comorbid mental health condition, such as depression or substance use disorders 